\title{
Evaluación de la ansiedad preoperatoria en pacientes sometidos a anestesia general. Estudio observacional.
}

\author{
Assessment of preoperative anxiety in patients under general \\ anesthesia. observational study.
}

\author{
Luisa F Leon-Girón ${ }^{*}$ \\ Jhoanna Betancourt Cañas ${ }^{1}$ \\ Claudia M Orozco-Chamorro ${ }^{2}$ \\ Ginna P. Cabra-Bautista ${ }^{3}$ \\ José A Calvache ${ }^{4}$
}

\author{
Recibido: 21 de noviembre de 2016 \\ Revisado: 10 de febrero de 2017 \\ Aceptado: 23 de marzo de 2017
}

\begin{abstract}
Resumen
Introducción: La ansiedad es una respuesta adaptativa del ser humano ante el peligro. El acto anestésico quirúrgico como generador de ansiedad se relaciona con mayor vulnerabilidad y difícil control del dolor postoperatorio, estancia hospitalaria prolongada, mayor tasa de accidentes anestésicos y vulnerabilidad a infecciones. Este estudio estimó la prevalencia de ansiedad preoperatoria en un grupo de pacientes sometidos a anestesia general mediante la escala de Ansiedad Preoperatoria y de Información de Ámsterdam (APAIS).

Métodos: Estudio observacional de corte transversal con pacientes que recibieron anestesia general en un hospital de III nivel de atención. Se obtuvo información sociodemográfica y clínica. Se determinó la prevalencia de ansiedad preoperatoria mediante la escala APAIS (utilizando un punto de corte de 11) y los potenciales factores asociados. Se realizó un análisis descriptivo y se calcularon odds ratios para las asociaciones. Además, se construyó un modelo de regresión logística para ajustar el efecto de las covariables en la presentación de ansiedad. Resultados: La prevalencia de ansiedad fue de 13\% [IC95\% 7\%-18\%]. Las mujeres, los pacientes ambulatorios, clasificación ASA I y sometidos a cirugía mayor sin antecedentes de procedimientos quirúrgicos previos presentaron una mayor frecuencia de ansiedad. La mayoría de los pacientes presentaban una baja necesidad de información $(76,8 \%)$. Tras el análisis ajustado, no se encontraron variables asociadas de forma importante a la presentación de ansiedad. Conclusiones: Se documentó una baja prevalencia de ansiedad y una baja necesidad de información por parte de los participantes. Se requiere continuar la investigación en el área en Colombia.
\end{abstract}

Palabras clave: Ansiedad, evaluación preoperatoria, Escala de Ansiedad Preoperatoria y de Información de Ámsterdam (APAIS), anestesia, procedimientos quirúrgicos menores.

\footnotetext{
${ }^{1}$ Médico general. Unidad de Epidemiología Clínica, Grupo de Investigación en Anestesiología GRIAN, Universidad del Cauca, Departamento de Anestesiología, Facultad de Ciencias de la Salud, Popayán, Cauca, Colombia.

${ }^{2}$ Estudiante de Medicina. Unidad de Epidemiología Clínica, Grupo de Investigación en Anestesiología GRIAN, Universidad del Cauca, Departamento de Anestesiología, Facultad de Ciencias de la Salud, Popayán, Cauca, Colombia.

${ }^{3} \mathrm{MD}$ Pediatra. Unidad de Epidemiología Clínica, Grupo de Investigación en Anestesiología GRIAN, Universidad del Cauca, Departamento de Anestesiología, Facultad de Ciencias de la Salud, Popayán, Cauca, Colombia.

${ }^{4}$ MD, MSc. Universidad del Cauca, Departamento de Anestesiología, Facultad de Ciencias de la Salud, Popayán, Cauca, Colombia, Department of Anesthesiology, Erasmus University Medical Centre Rotterdam, Rotterdam, The Netherlands.

*Correspondencia: Luisa F Leon-Girón. Correo electrónico: luisalenn19@hotmail.com
} 


\begin{abstract}
Introduction: Anxiety is an adaptive response of the human being to danger. The surgical anesthetic act is considered a generator of anxiety that is related to greater vulnerability and difficult control of postoperative pain, prolonged hospital stay, higher rate of anesthetic events and vulnerability to infections. This study aims to estimate the prevalence of preoperative anxiety in a group of patients undergoing surgery under general anesthesia using the Amsterdam Preoperative Anxiety and Information Scale (APAIS). Methods: A cross-sectional observational study performed with surgical patients who received general anesthesia at a third-level hospital. Sociodemographic and clinical information were obtained. Prevalence of preoperative anxiety was estimated by using the APAIS scale (cut of 11) and potential associated factors were evaluated. A descriptive analysis and crude odds ratios were calculated. In addition, a logistic regression model was constructed to adjust the effect of covariates. Results: Prevalence of anxiety was 13\% [IC95\% 7\%-18\%]. No variables were found to be significantly associated with the presence of anxiety. $76.8 \%$ of the participants had a low need for information. Women, outpatients, ASA I classification and patients under major surgery without a history of previous surgical procedures presented a higher frequency of anxiety. The majority of patients presented a low need for information (76.8\%). After the adjusted analysis, there were no variables related to the presentation of anxiety. Conclusions: A low prevalence of anxiety and a low need for information on the part of the participants were documented. It is necessary to do further research in this area in Colombia.
\end{abstract}

Key words: Anxiety, surgical clearance, preoperative anxiety, The Amsterdam Preoperative Anxiety and Information Scale (APAIS), anesthesia, minor surgical procedures. 


\section{Introducción}

La ansiedad es una respuesta adaptativa del ser humano ante el peligro. Proviene del latín "anxietas" y hace referencia a un estado de agitación, inquietud o zozobra del ánimo, que afecta al individuo de dos formas: como rasgo innato, resumido a una predisposición del comportamiento del individuo a percibir un amplio número de situaciones como amenazantes, que permanece en el tiempo; como estado cuando es un período transitorio de ansiedad, que es percibido por el individuo de manera consciente como tensión, aprehensión y elevada actividad del sistema nervioso autónomo ${ }^{(1,2)}$

Tomando como generador de ansiedad el acto anestésico quirúrgico, la ansiedad preoperatoria puede ser entendida como una respuesta de anticipación al daño ${ }^{(3)}$. Sin embargo, su importancia radica en que un nivel alto de ansiedad genera mayor vulnerabilidad y difícil control del dolor postoperatorio, se requiere mayor administración de analgesia e incluso prolonga la estancia hospitalaria, se relaciona directamente a mayor tasa de accidentes anestésicos y aumento de vulnerabilidad a infecciones ${ }^{(4-6)}$.

Existen diferentes escalas para determinar la presencia o no de ansiedad para el acto quirúrgico, dentro de las que se encuentran la escala STAI (State Trait Anxiety Inventory) que consiste en veinte preguntas y caracteriza la ansiedad como rasgo y como estado; es ampliamente utilizada sin embargo no tiene preguntas específicas hacia el acto quirúrgico ${ }^{(7)}$. De igual forma se han planteado otras escalas que abordan el acto anestésico como la Hospital Anxiety and Depresion Scale $(\text { HADS })^{(8)}$, la escala visual análoga ${ }^{(9)}$ y The Amsterdam Preoperative Anxiety and Information Scale (APAIS) que consta de seis preguntas, en donde cuatro están orientadas al procedimiento anestésico e intervención quirúrgica y dos hacia la demanda de información, por lo que se considera la más apropiada para determinar ansiedad preoperatoria ${ }^{(10)}$.

Según la Organización Mundial de la Salud (2011), anualmente más de 4 millones de pacientes se someten a cirugía en el mundo y se estima que del 50 al $75 \%$ desarrollan algún grado de ansiedad durante el periodo preoperatorio $^{(11)}$. En Latinoamérica la prevalencia de ansiedad preoperatoria oscila entre $33,3 \%$ y $75 \%$ en Perú ${ }^{(12,13)}$, en Ecuador del $60 \%$ para Anestesia general y raquídea ${ }^{(14)}$. En México se encuentran rangos entre $28 \%$ y $76 \%$ para anestesia regional ${ }^{(15)}$.

En Colombia se registran escasos datos de caracterización de ansiedad preoperatoria en pacientes adultos. En un estudio realizado con 294 pacientes programados para cirugía electiva en el Hospital Universitario del Caribe de Cartagena de Indias, se aplicó la escala APAIS encontrando una prevalencia de ansiedad preoperatoria de $36,1 \%{ }^{(16)}$. En Bogotá, se realizó un estudio con 21 pacientes que iban a ser sometidos a colecistectomía laparoscópica, utilizando la escala de ansiedad de Beck, encontrando que todos los pacientes intervenidos presentaron algún grado de ansiedad: leve en 7 , moderada en 11 y grave en $3^{(17)}$. Una tesis en proceso de publicación buscó validar la escala APAIS para facilitar la identificación de ansiedad preoperatoria, el $80 \%$ de los encuestados refirió entender las palabras usadas en los seis enunciados ${ }^{(18)}$.

En la literatura se reporta que la realización de consulta pre anestésica actúa como un factor protector respecto a la ansiedad preoperatoria, que la misma se presenta en mayor medida en las mujeres, aunque el grado de ansiedad disminuye cuando se ha tenido exposición previa a anestesia y cirugía y esta inversamente relacionada con el grado de escolaridad $^{(14,15,19,20)}$.

El objetivo de este estudio fue estimar la prevalencia de ansiedad preoperatoria en un grupo de pacientes sometidos a cirugía bajo anestesia general mediante la escala de Ansiedad Preoperatoria y de Información de Ámsterdam (APAIS) y explorar posibles determinantes en su presentación.

\section{Métodos}

Estudio observacional de corte transversal en el cual se incluyeron pacientes entre 18 y 70 años de edad atendidos en el servicio de cirugía del Hospital Universitario San José (HUSJ) de Popayán y sometidos a procedimientos bajo anestesia general durante un periodo de 4 meses (2015 - 2016), procedentes de los servicios de cirugía ambulatoria, hospitalización y urgencias.

Fueron excluidos los pacientes que requirieron traslado a la Unidad de Cuidado Intensivo o que provenían de ella, que requirieron ventilación mecánica postoperatoria o presentaron estados neurológicos o mentales que no hicieron posible la evaluación verbal del dolor en el servicio de Cirugía (Glasgow igual o menor de 14). También se excluyeron pacientes con emergencias vitales, todo procedimiento que implicase anestesia neuroaxial (caudal, epidural o subaracnoidea) o regional, pacientes con clasificación ASA igual o mayor a 4, no comprensión del idioma español y pacientes que no aprobaran ingresar al estudio. El protocolo del estudio fue aprobado previamente por el Comité de Ética del HUSJ y los pacientes manifestaron su consentimiento informado por escrito.

Para la recolección de información se empleó un instrumento elaborado por los investigadores que se constituía de cuatro apartados: 1) identificación y antecedentes (edad en años, género, peso, etnia, nivel educativo, clasificación ASA, servicio de procedencia ambulatorio, hospitalización o urgencias); 2) variables de admisión: grado de dolor mediante la escala numérica del dolor preoperatoria, cirugías previas, uso de medicamentos preoperatorios; 3) variables del procedimiento quirúrgico: localización anatómica de 
la cirugía y tipo de cirugía (clasificada como cirugía mayor y menor y considerando como mayor cualquier procedimiento localizado en cabeza, cuello, tórax y abdomen); y 4) Escala de Ansiedad APAIS, con seis ítems: dos que evalúan la ansiedad frente al procedimiento quirúrgico, dos frente al procedimiento anestésico y dos que representan la demanda de información. (Tabla 1$)^{(6)}$.

Tabla 1. Puntuación de Evaluación Preoperatoria "Amsterdam Preoperative Anxiety and Information Scale" (APAIS)

\begin{tabular}{|l|l|l|l|l|l|}
\hline \multicolumn{1}{|c|}{ Preguntas* } & $\mathbf{1}$ & $\mathbf{2}$ & $\mathbf{3}$ & $\mathbf{4}$ & $\mathbf{5}$ \\
\hline 1. Estoy inquieto con respecto a la anestesia & & & & & \\
\hline 2. Pienso continuamente en la anestesia & & & & & \\
\hline $\begin{array}{l}\text { 3. Me gustaría recibir una información lo } \\
\text { más completa posible con respecto a la anestesia }\end{array}$ & & & & & \\
\hline 4. Estoy inquieto con respecto a la intervención & & & & & \\
\hline 5. Pienso continuamente en la intervención & & & & & \\
\hline $\begin{array}{l}\text { 6. Me gustaría recibir una información lo más } \\
\text { completa posible con respecto a la Intervención }\end{array}$ & & & & & \\
\hline
\end{tabular}

* Estas afirmaciones deben calificarse en una escala likert de cinco puntos, donde 1 representa en desacuerdo y cinco extremadamente de acuerdo. Adicionalmente, cuenta con tres subescalas: 1) Ansiedad relacionada con la anestesia $(\mathrm{A}=1+2), 2)$ Ansiedad relacionada con la cirugía ( $\mathrm{S}=4+5)$ y 3$)$ Necesidad de información $(\mathrm{NI}=3+6)$. La puntuación total combinada de ansiedad se calcula $(\mathrm{C}=\mathrm{A}+\mathrm{S})$.

Fuente: Modificado por los autores (10)

El desenlace primario en estudio fue la prevalencia de ansiedad preoperatoria evaluada mediante la escala APAIS. Estudios latinoamericanos han mostrado prevalencias de ansiedad preoperatoria en rangos desde $28 \%$ a $75 \%(12,15)$. Con un estimado para la prevalencia de ansiedad del 50\%, una precisión de $8 \%$ y una confianza al $95 \%$ se estimó un tamaño de muestra de 150 pacientes. Con los datos se construyó una base de datos en Microsoft Excel.

Se realizó un análisis descriptivo y gráfico utilizando para variables cuantitativas medidas de tendencia central y de dispersión (media, mediana, desviación estándar, rango intercuartílico) y para variables cualitativas frecuencias absolutas y proporciones. La estimación de la prevalencia de ansiedad se calculó utilizando corte de 11 puntos en la escala APAIS y se acompañó con el cálculo de sus intervalos de confianza al 95\% (IC95\%). El punto de corte fue escogido debido a que se estima que con punto de corte de 11 se obtiene buena sensibilidad y especificidad, el valor predictivo positivo (VPP) es de $71 \%$ y los falsos positivos de $9 \%$, con punto de corte 13 los resultados son similares sin embargo aumenta el VPP y por consiguiente disminuye el valor de falsos positivos. El punto de corte de 10 incrementa la sensibilidad pero disminuye la especificidad por lo que el VPP disminuye significativamente y resulta en un alto número de falsos positivos ${ }^{(1)}$.

Se exploró de forma cruda la influencia de las variables género, edad, procedencia, nivel educativo, antecedente quirúrgico y tipo de cirugía en la presentación de ansiedad. Se utilizó la prueba exacta de Fisher o el test de chi2 de acuerdo a las frecuencias mínimas en cada categoría. Se calculó el odds ratio (OR) crudo para las asociaciones y sus intervalos de confianza al $95 \%$. Posteriormente se construyó un modelo de regresión logística para ajustar el efecto de las covariables en la presentación de ansiedad. Dado el limitado número de eventos en el desenlace se optó únicamente el corte de 11 para la definición de "ansiedad". Para evaluar el ajuste del modelo se utilizó el R2 y se hizo análisis de los residuos. Para todos los análisis se prefijó un nivel de confianza de 0,05 pero se reportan los efectos absolutos de las intervenciones. Todos los análisis se realizaron en R y R Studio ${ }^{(21)}$.

El protocolo del estudio fue aprobado previamente por el Comité de Ética del HUJS en agosto de 2014 y los pacientes manifestaron su consentimiento informado por escrito.

\section{Resultados}

Se incluyeron 151 pacientes de los cuales $55 \%(\mathrm{n}=83)$ fueron del género femenino, presentaron una media de edad de $40.45 \pm 14.72$, pertenecieron a la raza mestiza en un $96 \%$, el nivel educativo predominante fue de primaria en $49 \%(n=75)$ de los entrevistados. En cuanto al servicio de procedencia, $53 \%(\mathrm{n}=80)$ de los pacientes fueron del servicio ambulatorio, $45 \%(n=68)$ había tenido una cirugía previa y $63 \%(\mathrm{n}=95)$ fueron clasificados como ASA I. $80 \%(n=121)$ de los participantes fueron sometidos a cirugía mayor y $33.8 \%$ $(\mathrm{n}=51)$ presentaron dolor severo preoperatorio. (Tabla 2).

La ansiedad preoperatoria se encontró en 19 pacientes (13\%) [IC95\% 7-18], con un punto de corte en la escala de APAIS de 11. Utilizando el punto de corte de 13 en la escala fue de 3\% [IC 95\% 0-5]. Las mujeres resultaron más ansiosas que los hombres, así como los pacientes que eran ambulatorios, con clasificación de ASA I, sometidos a cirugía mayor sin antecedentes de 
procedimientos quirúrgicos previos (Tabla 2).

La Tabla 2 presenta el análisis de variables en estudio y la presentación de ansiedad preoperatoria. No se encontraron factores asociados de forma importante a una mayor probabilidad de ansiedad en la población estudiada.

Tabla 2. Análisis crudo y ajustado de la influencia de variables en la prevalencia de ansiedad preoperatoria $(\mathrm{n}=151)$.

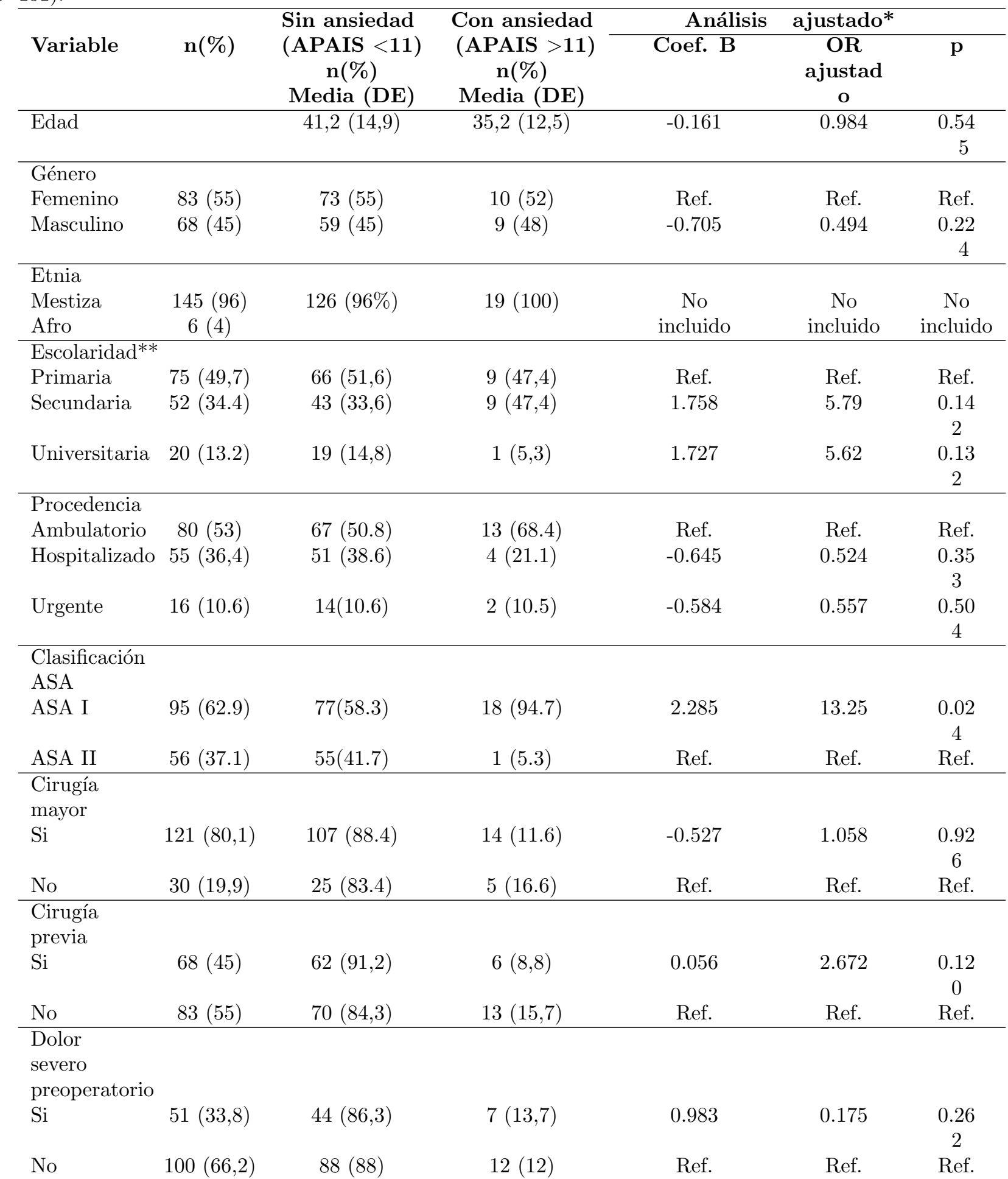

* Variables incluidas en el modelo: género, edad, procedencia, nivel educativo, antecedente quirúrgico y tipo de cirugía.

** Datos perdidos en variable escolaridad Fuente: Elaborado por autores.

Al desglosar los niveles de información anestésicoquirúrgica, evaluadas con las preguntas 3 y 6 del cuestionario APAIS: "Me gustaría recibir una información lo más completa posible con respecto a la anestesia" y "Me gustaría recibir una información lo más completa posible con respecto a la intervención", se encontró que 
116 pacientes $(76,8 \%)$ requerían información adicional acerca del procedimiento quirúrgico y/o anestésico al cual se sometían; $28(18.5 \%)$ requerían información promedio y 7 (4,6\%) información detallada. La necesi- dad de información presentó una correlación positiva con la puntuación total de la escala de APAIS $(r=0,62)$ (Figura 1).

Figura 1. Correlación entre necesidad de información y la puntuación total de la escala de APAIS (n=151)

Figura 1.

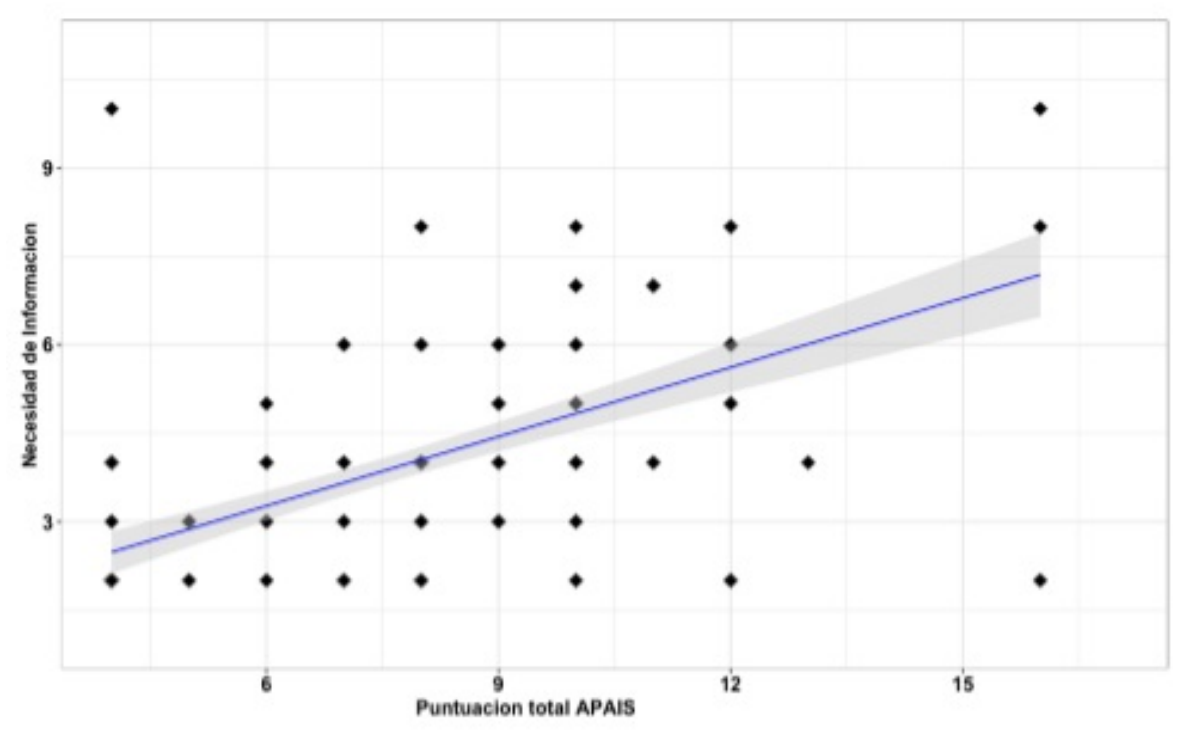

Fuente: Elaborado por autores.

\section{Discusión}

Los principales resultados de este estudio son: i) la prevalencia de ansiedad preoperatoria fue baja (13\%) utilizando el corte de 11 puntos en la escala APAIS. ii) se encontró una baja necesidad de información por parte de los pacientes con respecto al procedimiento quirúrgico y anestésico. iii) se encontró variables asociadas de forma importante a la probabilidad de ocurrencia de ansiedad preoperatoria.

La presencia de ansiedad preoperatoria es un acontecimiento frecuente, poco evaluado en los pacientes que serán sometidos a una intervención quirúrgica. Es un evento de gran importancia que se ha relacionado con diversos desenlaces adversos en el intra y posoperatorio. La prevalencia documentada en este estudio es baja en comparación a lo encontrado en diferentes estudios realizados en Quito - Ecuador ${ }^{(14)}$, Cartagena - Colombia ${ }^{(16)}$, Ciudad de Obregon - México ${ }^{(15)}$, Estado de México ${ }^{(19)}$ y Sao Paulo - Brasil ${ }^{(22)}$. En estos estudios se describieron frecuencias más altas de ansiedad con la escala APAIS, que oscilaron entre el $28 \%$ y el $76 \%$. Diversos autores atribuyen estos resultados a una falta de conocimiento e información por parte de pacientes previo al acto anestésico y quirúrgico.

Un reciente estudio colombiano presentó una evaluación de ansiedad mediante el uso de la Escala de ansiedad de Beck. Reportaron una frecuencia de ansiedad de $100 \%$ de los pacientes valorados y adicionalmente la categorizaron como leve, moderada o grave. Sin embargo, este estudio no presenta intervalos de confianza de la estimación de esta prevalencia, el cual asumimos es muy amplio dado el bajo número de sujetos evaluados y reportados por sus autores (21 pacientes $)^{(17)}$.

La escala APAIS consiste en una serie de preguntas con un valor de 1 a 5 puntos para cada respuesta, con un máximo de 20 puntos. Cuenta con una subescala de información, en donde obtener una puntuación entre 5 y 7 indica que los pacientes no requieren información y una calificación entre 8 a 10 indica que los pacientes requieren información más completa con el propósito de disminuir la ansiedad creada por el procedimiento quirúrgico o el acto anestésico. En un estudio ecuatoriano se observó una asociación directamente proporcional entre demanda de información y ansiedad, además que aquellos pacientes que reciben una información adecuada disminuyen los niveles de $\operatorname{ansiedad}^{(14)}$. Nuestros resultados apoyan la existencia de correlación entre la necesidad de información y el grado de ansiedad estimado. Por supuesto dicha correlación no es causal ${ }^{(23,24)}$.

Se ha descrito que la visita pre anestésica se comporta como un factor protector respecto a la ansiedad, relacionado con la mejor información que recibe el paciente acerca del procedimiento y la anestesia en esta intervención ${ }^{(14,15,22)}$. Cabe resaltar que este estu- 
dio no profundiza en este aspecto y, por el contrario, proporciona nuevas hipótesis como: La baja prevalencia de ansiedad encontrada representa ¿alta información previa?, ¿apropiada consulta preanestésica?, ¿desconocimiento total del proceso por parte de los pacientes?

En cuanto a las variables relacionadas con la presencia de mayor ansiedad preoperatoria los resultados son diversos. En estudios llevados a cabo en México y Sri Lanka encontraron que los pacientes de género femenino ${ }^{(10,15,26)}$, que tienen un grado de escolaridad mayor $^{(15)}$ y que no han experimentado una cirugía o anestesia previamente ${ }^{(26)}$ presentan mayor ansiedad; en otros estudios no se encontraron variables sociodemográficas con una relación significativa en los niveles de ansiedad al igual que en este ${ }^{(14,22)}$.

En nuestro estudio la presencia de ansiedad respecto al género, escolaridad y variables sociodemográficas concuerda con lo descrito en la literatura. Adicionalmente, la frecuencia de ocurrencia de ansiedad preoperatoria fue mayor en pacientes sanos ASA I, procedentes del servicio ambulatorio y con antecedente quirúrgico previo. La gran mayoría de los pacientes estudiados fueron ambulatorios y tenían consulta pre anestésica previa.

Dentro de las limitaciones de este estudio resaltamos su aplicación en un solo centro hospitalario, de modo que los resultados no pueden ser ampliamente generalizados. Además del tamaño de muestra relativamente pequeño y el bajo poder estadístico del estudio para establecer asociaciones con la frecuencia del evento. Adicionalmente, se documentó un bajo porcentaje de pacientes ansiosos con escolaridad universitaria, correspondientes sólo al 5,3\% de la población ansiosa lo cual dificulta las estimaciones. El limitado número de sujetos con el desenlace, al igual que algunas categorías de las covariables, evita desarrollar modelos multivariantes robustos para ajustar las potenciales asociaciones. Por otra parte, los pacientes procedentes del servicio de urgencias o aquellos clasificados con un ASA II o superior es reducido y esto se podría generalizar a otras poblaciones y explicar en parte la baja incidencia de ansiedad.

\section{Agradecimientos}

Responsabilidades éticas: Los autores declaran que se han seguido los protocolos éticos sobre la publicación de datos de pacientes, así como la garantía de que no aparecen datos personales de pacientes en este artículo. Apoyo financiero y patrocinio: Los autores declaran no haber recibido fondos para la preparación de este artículo. Conflictos de interés: Los autores no tienen conflicto de intereses para divulgar Presentación: ninguna.

\section{Bibliografía}

1. Spielberger CD. The theory and research on anxi- ety. In: Anxiety and behavior. 1966

2. Sierra JC, Ortega V, Zubeidat I. Ansiedad, Angustia Y Estrés: Tres Conceptos a Diferenciar. Rev Mal-Estar E Subjetividade / Fortaleza. 2003; 3(1): $10-59$.

3. Thomas V, Heath M, Rose D, Flory P. Psychological characteristics and the effectiveness of patientcontrolled analgesia. Br J Anaesth. 1995; 74(3):271-6. https://doi.org/10.1093/bja/74.3.271

4. Anderson KO, Masur FT. Psychological preparation for invasive medical and dental procedures. J Behav Med. 1983; 6(1):1-40.

5. Pérez Díaz R, Martín Carbonell M, Quiñones Castro M. Influencia de la ansiedad prequirúrgica en la evolución de la cirugía de las varices. Rev Cuba Angiol y Cir Vasc. 2000; 1(2):95-100.

6. Boker A, Brownell L, Donen N. The Amsterdam preoperative anxiety and information scale provides a simple and reliable measure of preoperative anxiety. Can J Anesth. 2002; 49(8):792-8. https://doi.org/10.1007/BF03017410

7. Spielberger CD, Gorsuch RL, Lushene RE. StateTrait Anxiety Inventory (STAI). Manual for the StateTrait Anxiety Inventory. 2010.

8. Zigmond AS, Snaith RP. The Hospital Anxiety and Depression Scale. Acta Psychiatr Scand. 1983;

9. Kindler CH, Harms C, Amsler F, Ihde-Scholl T, Scheidegger $\mathrm{D}$. The visual analog scale allows effective measurement of preoperative anxiety and detection of patients' anesthetic concerns. Anesth Analg. 2000; $90(3): 706-12$.

10. Moerman N, Van Dam FSAM, Muller MJ, Oosting H. The Amsterdam Preoperative Anxiety and Information Scale (APAIS). Anesth Analg. 1996; (3):445-51.

11. Quijano SCDP. Nivel de ansiedad del paciente en el preoperatorio en el servicio de cirugía del Hospital Nacional Dos de Mayo - 2013. Univ Nac Mayor San Marcos. 2014;

12. Gonzales Butamante G, Pinto Samanez F. Escala Amsterdam Ansiedad Escala Informacion Apais Fase Preoperatoria Peru 2005. Universia.net. 2010.

13. Torres Treviños I. Ansiedad preoperatoria en pacientes programados para cirugía Centro quirúrgico Clínica el Golf Lima-Perú 2014. [Internet]. Universidad Nacional Mayor de San Marcos (Lima); 2014. Available from: http://hdl.handle.net/123456789/5144 
14. Naranjo KF, Salazar JP. Comparación del nivel de ansiedad en los pacientes de cirugía programada mediante la aplicación de la escala de ansiedad e infromación preoperatoria de Amsterdam realizada en la visita pre anestésica y comparada con grupo control en los hospitales Eugenio. 2012;12-55. Available from: http://www.dspace.uce.edu.ec/bitstream/25000/1113 /1/T-UCE-0006-39.pdf

15. Valenzuela-Millán J, Barrera-Serrano JR, OrnelasAguirre JM. Ansiedad preoperatoria en procedimientos anestésicos. Cir Cir. 2010; 78:151-156.

16. Muñoz Pérez X. Factores de riesgo asociados a la ansuedad preoperatoria en cirugias electivas en el Hospital Universitario del Caribe de Cartagena, Colombia. Universidad de Cartagena; 2018.

17. Quintero A, Yasnó D, Riveros O, Castillo J, Borráez B. Ansiedad en el paciente prequirúrgico: un problema que nos afecta a todos. Rev Colomb Cir. 2017; 32:115-20.

18. Pinzón Bayona MY, Pastrana Londoño OA. Validación de la Escala de Información de ansiedad preoperatoria de Amsterdam (APAIS) a un Caso Colombiano [Internet]. Universidad del Rosario; $2017 . \quad$ Available from: http://repository.urosario.edu.co/handle/10336/12853

19. Omar K, Armenta H, Virgen R, Morales P. Ansiedad preoperatoria. Medicine (Baltimore). 2014;
20. Salamanca Ragua N. Evaluación preanestésica. Rev la Fac Ciencias la Salud Univ del Cauca [Internet]. 2005; 7(2): 33-39.

21. R Development Core Team RFFSC. R: A Language and Environment for Statistical Computing. Vienna Austria R Found Stat Comput. 2008;

22. Kiyohara LY, Kayano LK, Oliveira LM, Yamamoto MU, Inagaki MM, Ogawa NY, et al. Surgery information reduces anxiety in the pre-operative period. Rev Hosp Clin Fac Med Sao Paulo. 2004; 59(2):51-6.

23. Altman N, Krzywinski M. Points of Significance: Association, correlation and causation. Nat Methods. 2015

24. Schober P, Boer C, Schwarte LA. Correlation Coefficients: Appropriate Use and Interpretation. Anesth Analg. 2018; 26(5):1763-1768. doi: 10.1213/ANE.0000000000002864.

25. Jawaid M, Mushtaq A, Mukhtar S, Khan Z. Preoperative anxiety before elective surgery. Neurosciences (Riyadh). 2007; 12(2):145-8.

26. Matthias AT, Samarasekera DN. Preoperative anxiety in surgical patients - Experience of a single unit. Acta Anaesthesiol Taiwanica. 2012; 50(1):3-6. doi: 10.1016/j.aat.2012.02.004. 